\title{
Prevalence of anxiety and depressive symptoms in men with erectile dysfunction
}

K Pankhurst, MB ChB

G Joubert, BA, MSC

P J Pretorius, MB ChB, MMed (Psych)

Departments of Psychiatry and Biostatistics,

University of the Free State, Bloemfontein

Objectives. To determine the prevalence of anxiety and depressive symptoms in men presenting to a sexual dysfunction clinic in Bloemfontein with erectile dysfunction (ED); to determine the relationship, if any, between age and mood/anxiety symptoms in such patients; and to make clinicians aware of the co-morbidity of anxiety/mood symptoms and ED.

Methods. An observational analytical study was undertaken of 100 consecutive male patients of all ages presenting with ED (with a score less than 20 on the 5-item intensity scale for EDI. Age, race, marital and employment status were noted as well as social habits including smoking and alcohol use. The presence of known medical conditions and surgical procedures was ascertained. All current prescription medication was recorded. Panic disorder, obsessive-compulsive disorder, generalised anxiety disorder and social phobia were rated using the Mini International Neuropsychiatric Interview, while the Hamilton Rating Scale for Depression was used to rate depressive symptoms.

Results. Thirty-three per cent of respondents had depressive symptoms, and of this group 36\% had a co-morbid anxiety disorder. In total, $21 \%$ of patients had an anxiety disorder. Anxiety disorders were more common with moderate to severe ED. No anxiety disorders occurred in patients with mild ED. The majority of participants suffering from severe ED were evenly spread in age from 30 to 69 years. Participants suffering from moderate to severe ED were more likely to have medical conditions, most notably hypertension.

Conclusion. The results of this study suggest that men suffering from ED are likely to have a co-morbid psychiatric disorder (42\%), with the prevalence of depressive symptoms (33\%) and anxiety disorders (21\%) being higher than in the general population. Significant concomitant medical conditions (most notably hypertension) were more common in men with moderate to severe ED.

Erectile dysfunction (ED) is defined as the consistent inability to achieve and/or maintain an erection sufficient for satisfactory sexual performance, over a 3-month period.' ED is a common sexual disorder with a prevalence rate of $40 \%$ among 40 -yearold men, increasing to $70 \%$ among 70 -year-olds. ${ }^{2,3}$ There is a high correlation of depression and anger symptoms in patients suffering from ED. ${ }^{2}$ The relationship between ED and depressive illness is still unclear, with general acceptance that the aetiology of ED is in most cases multifactorial in origin with a strongly related organic component.'

The Massachusetts Male Aging Study ${ }^{3}$ showed a significant association between ED and age, diabetes mellitus, heart disease and hypertension. The same study showed that patients with depression have a 1.82 times higher chance of developing moderate to severe ED than patients not suffering from depression. ${ }^{3,4}$ The triad of ED, cardiovascular disease and depression are likely to increase the morbidity and mortality rate in such patients. ${ }^{5}$ Four models of cause-and-effect association have been proposed to explain the complex relationship between depression and ED: 6

Model 1. The primary illness is that of depression, with ED a symptom of the depressive disorder. 
Model 2. ED is the primary illness, with loss of sexual function possibly leading to depressive symptoms.

Model 3. Medical conditions, e.g. hypothyroidism, can result in both ED and depressive symptoms.

Model 4. Depression and ED may be coincidentally comorbid. Although prevalence rates of ED and depression increase with age, they may be co-morbid in age groups other than the elderly.

However, sexual dysfunction is a common side-effect of antidepressant treatment, and may also explain the relationship between depression and ED. Serotonin reuptake inhibitors (SRIs), serotonin noradrenalin re-uptake inhibitors and tricyclic antidepressants are known to impact on sexual functioning loften leading to poor compliance and patient dissatisfaction). SRI-induced sexual dysfunction occurs in $30-70 \%$ of patients treated for depression, while buproprion and nefazodone are associated with the least sexual dysfunction.? Antidepressant-induced sexual dysfunction may lead to premature discontinuation of treatment, raising the risk of relapse and recurrence of major depression.

There is also a high prevalence of anxiety in patients with $E D,{ }^{8}$ and performance anxiety has been emphasised as a major cause of psychogenic ED, ${ }^{9}$ with the effects on sexual arousal in men having been shown to be mediated largely by cognitive factors. ${ }^{9}$ Two studies reported that approximately one-third of men with ED have a co-morbid psychiatric disorder, ${ }^{6}$ predominantly affective disorder. Even when the full diagnostic criteria are not met, there are high levels of anxiety and depressive symptoms. ${ }^{\circ}$ Thus ED should be considered a multifactorial condition requiring a multidisciplinary approach and management.

The aim of the present study was to determine the prevalence of anxiety and depressive symptoms in men presenting with ED at the Men's Clinic International in Bloemfontein. Further aims were to determine the relationship, if any, between the severity of ED and mood/anxiety symptoms. We also hope to increase awareness of the co-morbidity of anxiety/mood symptoms and ED.

\section{Material and methods}

The study was an observational analytical study of 100 consecutive male patients of all ages presenting to the Men's

\section{International Index of Erectile Function (IIEF)'}

\section{Over the past 4 weeks:}

1 How do you rate your confidence that you could get and keep an erection?

$\begin{array}{ccccc}\text { Very Low } & \text { Low } & \text { Moderate } & \text { High } & \text { Very High } \\ 1 & 2 & 3 & 4 & 5\end{array}$

2 When you had erections with sexual stimulation, how often were your erections hard enough for penetration?

$\begin{array}{cccccc}\begin{array}{c}\text { No Sexual } \\ \text { Activity }\end{array} & \begin{array}{c}\text { Almost never } \\ \text { or never }\end{array} & \begin{array}{c}\text { A few times } \\ \text { (less than } 1 / 2 \text { the time) }\end{array} & \begin{array}{c}\text { Sometimes } \\ \text { (112 the time) }\end{array} & \begin{array}{c}\text { Most times } \\ \text { (more than } 1 / 2 \text { the time) }\end{array} & \begin{array}{c}\text { Almost always } \\ \text { or always }\end{array} \\ \mathbf{0} & \mathbf{1} & \mathbf{2} & \mathbf{3} & \mathbf{4} & \mathbf{5}\end{array}$

5 During sexual intercourse, how often were you able to maintain your erection after you had penetrated (entered) your partner?

Did not attempt Almost never A few times Sometimes intercourse or never (less than 1/2 the time) (11/2 the time) (more than 1/2 the time) or always

$\begin{array}{llllll}0 & 1 & 2 & 3 & 4 & 5\end{array}$

4 During sexual intercourse, how difficult was it to maintain your erection to completion of intercourse?

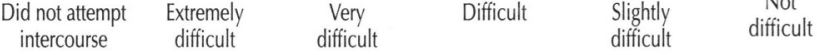

$\begin{array}{lllllll}0 & 1 & 2 & 3 & 4 & 5\end{array}$

5 When you attempted sexual intercourse, how often was it satisfactory for you?

Did not attempt Almost never A few times Sometimes Most times Almost always intercourse or never (less than $1 / 2$ the time) (1/2 the time) (more than 1/2 the time) or always

$\begin{array}{lllllll}0 & 1 & 2 & 3 & 4 & 5\end{array}$

\begin{tabular}{|l|l|}
\hline Score & Interpretation \\
\hline $22-25$ & No ED \\
\hline $17-21$ & Mild ED \\
\hline $12-16$ & Mild - Moderate ED \\
\hline $8-11$ & Moderate ED \\
\hline $5-7$ & Severe ED \\
\hline
\end{tabular}

Patient Name:

Score

Reference: 1. Rosen RC, Cappelleri IC, et al. Development and evaluation of an abridged 5 version of the Internation
Index of Erectile Function (ill -5 ) as a diagnostic tool for erectile dysfunction. Int I Impotence Res $1999 ; 11: 319-32$.

Fig. 1. International Index of Erectile Function. ${ }^{10}$

Clinic International in Bloemfontein, South Africa with ED. The Ethics Committee, Faculty of Health Sciences, of the University of the Free State approved the protocol for the study, and all study participants gave written informed consent.

Patients who did not give permission for the study and those who scored 20 or more on the 5-item intensity scale for ED were excluded from the study.

ED was scored using the International Index of Erectile Function ${ }^{10}$ (Fig. 1) as follows: 5 - 10 severe, 11 - 15 moderate, $16-20$ mild, and 21 - 25 normal.

The patient completed the self-administered 5-item intensity scale before undergoing a structured interview with the first author. The scale was part of the standard information requested from all patients at the clinic and was discussed with the patient at the beginning of the interview. Age, race, marital 
and employment status were noted as well as social habits including smoking and alcohol use. The presence of known medical conditions such as psychiatric disorders, hypertension, diabetes, cholesterol and cardiovascular disease was ascertained through questioning by the interviewer. Surgical procedures were noted and the type of surgery documented. The interviewer recorded all current prescription medication, noting the subject's stated reason for use of each.

Hypertension at baseline was indicated if one or more of the following four conditions were met, viz.: (i) the subject reported being treated for high blood pressure; (ii) history-taking revealed use of antihypertensive medication; (iii) the subject's systolic blood pressure was greater than or equal to $140 \mathrm{mmHg}$; or (iv) the subject's diastolic blood pressure was greater than or equal to $90 \mathrm{mmHg}$. Diabetes mellitus was indicated if the subject reported being treated for diabetes mellitus, if history-taking revealed use of medication for diabetes mellitus, or if a random blood glucose level of $>11.1$ was found. Premature ejaculation was defined as the persistent or recurrent achievement of orgasm and ejaculation before the desired time period. ${ }^{11}$

The above procedures are routine during the assessment of patients at the clinic and only the psychiatric rating scales were additional. Following assessment and diagnosis the patient was started on the relevant therapy.

Baseline depressive symptomatology was measured using the 17-item Hamilton Rating Scale for Depression (HAM-D). ${ }^{12}$ The HAM-D was developed as a measure of treatment outcome, but it is commonly used as a screening scale. The HAM-D is a 17 item rating scale used to systematise clinical observations of features related to depression. The total score for each individual was classified as minimal $(0-7)$, mild (8 - 17), moderate $(18-25)$ and severe $(\geq 26)$.

Panic disorder, obsessive-compulsive disorder, generalised anxiety disorder and social phobia were rated using the Mini International Neuropsychiatric Interview $(\mathrm{MINI}){ }^{13}$ During the research it was observed that the questions asked on the MINI rating scales were too direct for some of the clients and falsepositive answers were obtained. Non-direct questions were adopted and if a positive answer was obtained more direct questions from the MINI rating scale were asked to confirm the answer.

Results were summarised as frequencies and percentages (categorical variables) and means, standard deviations or percentiles (numerical variables). Associations were assessed by risk differences with $95 \%$ confidence intervals (Cls).

\section{Results}

Selected characteristics of the respondents are listed in Table I. The majority (79\%) were in the 30 - 59-year age group. The majority were black (72\%), married (75\%) and employed $(77 \%)$. Just under half of the respondents were active cigarette smokers $(46 \%)$ and $70 \%$ reported consuming alcohol.

\section{Table I. Characteristics of respondents $(N=100)$}

\begin{tabular}{lc}
\hline & $\%$ \\
\hline Age (yrs) & 6 \\
$20-29$ & 22 \\
$30-39$ & 29 \\
$40-49$ & 28 \\
$50-59$ & 11 \\
$60-69$ & 4 \\
$70-79$ & \\
Race & 28 \\
White & 72 \\
Black & 0 \\
Other & \\
Marital status & 14 \\
Never married & 75 \\
Currently married & 8 \\
Divorced & 3 \\
Widowed & 77 \\
Employed & 46 \\
Smoking currently & 70 \\
Current alcohol use & \\
\hline
\end{tabular}

Table II shows the severity of ED, risk factors for ED and comorbid psychiatric diagnosis. The majority of respondents had severe ED (57\%); 36\% had moderate ED, and $7 \%$ mild ED. More than half of all respondents (60\%) reported premature ejaculation.

Mild depressive symptoms were noted in 15 patients. A further 15 respondents had moderate depressive symptoms and 3 had severe depressive symptoms (Table II). No psychiatric disorders were found in $58 \%$ of the respondents and 8 respondents only had mild depressive symptoms (Fig. 2). Thirteen respondents had moderate to severe depressive symptoms and no other psychiatric disorder. Twelve respondents had both depressive symptoms and an anxiety disorder. None of the patients with cardiovascular disease scored more than 17 on the HAMD. Eight patients fulfilled the criteria for panic disorder without 


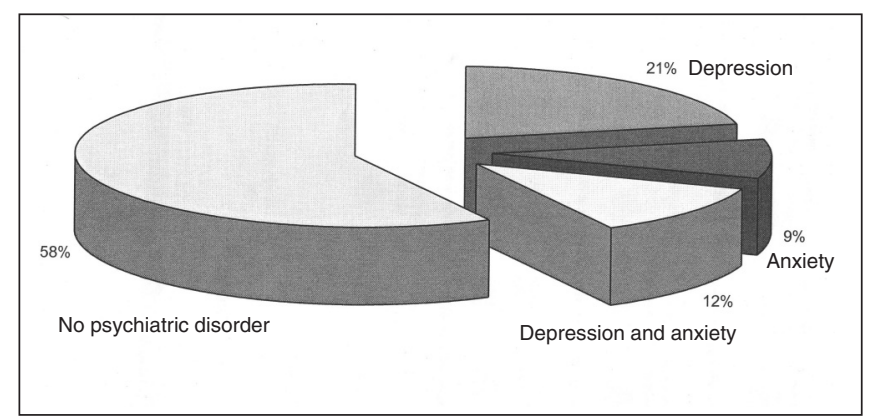

Fig. 2. Erectile dysfunction and co-morbid psychiatric disorders.

Table II. Severity and risk factors for erectile dysfunction and co-morbid psychiatric diagnosis $(N=100)$

\begin{tabular}{|c|c|c|}
\hline & \multicolumn{2}{|c|}{$\begin{array}{l}\text { Percentage of } \\
\text { patients }\end{array}$} \\
\hline \multicolumn{3}{|l|}{$\overline{\mathrm{ED}}$} \\
\hline Mild (IIEF score $16 \quad 20)$ & 7 & \\
\hline Moderate (IIEF score 11 15) & 36 & \\
\hline Severe (IIEF score 0 10) & 57 & \\
\hline Premature ejaculation & 60 & \\
\hline Previous treatment for ED & 10 & \\
\hline \multicolumn{3}{|l|}{ HAM D score } \\
\hline 07 & 67 & \\
\hline 817 & 15 & \\
\hline $18 \quad 25$ & 15 & \\
\hline$>25$ & 3 & \\
\hline Panic disorder with agoraphobia & 0 & \\
\hline Panic disorder without agoraphobia & 8 & \\
\hline Agoraphobia & 0 & \\
\hline Obsessive compulsive disorder & 2 & \\
\hline Generalised anxiety disorder & 9 & \\
\hline \multirow[t]{2}{*}{ Social phobia } & 7 & \\
\hline & & $\begin{array}{l}\text { Currently } \\
\text { on } \\
\text { treatment }\end{array}$ \\
\hline Medical conditions & & $(\%)$ \\
\hline Psychiatric disorder & 2 & 100 \\
\hline Hypertension & 41 & 78 \\
\hline Diabetes & 17 & 82 \\
\hline Cholesterol & 10 & 80 \\
\hline Cardiovascular disease & 5 & 80 \\
\hline \multicolumn{3}{|l|}{ Operations } \\
\hline Coronary artery bypass grafts & 2 & \\
\hline Stent & 2 & \\
\hline Prostatectomy & 3 & \\
\hline Pelvic & 0 & \\
\hline Vascular & 1 & \\
\hline IIEF = International Index of Erectile Function..$^{10}$ & & \\
\hline
\end{tabular}

agoraphobia, ${ }^{9}$ for generalised anxiety disorder ${ }^{7}$ and for social phobia. In total, 21 patients had an anxiety disorder. The presence of an anxiety disorder tended to correlate with moderate to severe ED, but not with mild ED.

Under medical conditions $41 \%$ of participants suffered from hypertension, 17\% from diabetes mellitus, 10\% from hypercholesterolaemia and 5\% from cardiovascular disease. Only $2 \%$ of patients reported being treated for a psychiatric condition. The majority of these respondents were receiving medication for the relevant conditions. With regard to surgery, 2 respondents had coronary artery bypass grafts, 2 had stents inserted and 3 respondents had had prostatectomy performed in the past.

Table III compares the respondents with mild $(N=7)$, moderate $(N=36)$ and severe $(N=57) E D$ in terms of age, race and marital status. The majority of the participants suffering from severe ED were evenly spread from the ages of 30 to 69 years. Moderate ED was mainly reported in the 40 - 59-year age groups. Almost $60 \%$ of the participants with mild ED were aged between 40 and 49 years.

Table III. Comparison of characteristics and severity of erectile dysfunction

\begin{tabular}{lccc}
\hline & $\begin{array}{l}\text { Mild } \\
(\%)\end{array}$ & $\begin{array}{l}\text { Moderate } \\
(\%)\end{array}$ & $\begin{array}{l}\text { Severe } \\
(\%)\end{array}$ \\
& $(N=7)$ & $(N=36)$ & $(N=57)$ \\
\hline Age (yrs) & & & \\
2029 & 14 & 3 & 7 \\
3039 & 14 & 22 & 23 \\
$40 \quad 49$ & 58 & 30 & 25 \\
50 59 & 14 & 39 & 23 \\
60 69 & 0 & 3 & 17 \\
7079 & 0 & 3 & 5 \\
Race & & & \\
White & 29 & 22 & 32 \\
Black & 71 & 78 & 68 \\
Marital status & & & \\
Never married & 14 & 8 & 18 \\
Currently married & 86 & 81 & 70 \\
Divorced & 0 & 11 & 7 \\
Widowed & 0 & 0 & 5 \\
Employed & 100 & 81 & 72 \\
Smoking & 57 & 56 & 39 \\
Alcohol use & 86 & 69 & 68 \\
\hline
\end{tabular}

Table IV compares the severity of ED with risk factors and comorbid psychiatric diagnosis. Participants suffering from moderate to severe ED were more likely to have medical conditions, most notably hypertension. Two-thirds of those 
Table IV. Comparison of the severity of erectile dysfunction and risk factors

\begin{tabular}{|c|c|c|c|}
\hline & $\begin{array}{l}\text { Mild } \\
(\%) \\
(N=7)\end{array}$ & $\begin{array}{l}\text { Moderate } \\
(\%) \\
(N=36)\end{array}$ & $\begin{array}{l}\text { Severe } \\
(\%) \\
(N=57)\end{array}$ \\
\hline \multicolumn{4}{|l|}{ Medical conditions } \\
\hline Psychiatric disorders & 0 & 3 & 2 \\
\hline Hypertension & 14 & 33 & 49 \\
\hline Diabetes & 14 & 19 & 16 \\
\hline Cholesterol & 0 & 11 & 11 \\
\hline $\begin{array}{l}\text { Cardiovascular } \\
\text { disease }\end{array}$ & 0 & 8 & 4 \\
\hline $\begin{array}{l}\text { Previous treatment } \\
\text { for } E D\end{array}$ & 0 & 6 & 14 \\
\hline \multicolumn{4}{|l|}{ Operations } \\
\hline CABG & 0 & 3 & 2 \\
\hline Stent & 0 & 6 & 0 \\
\hline Prostatectomy & 0 & 6 & 2 \\
\hline Pelvic & 0 & 0 & 0 \\
\hline Vascular & 0 & 3 & 0 \\
\hline \multicolumn{2}{|l|}{ Premature ejaculation 71} & 72 & 51 \\
\hline $\begin{array}{l}\text { Panic disorder } \\
\text { with agoraphobia }\end{array}$ & 0 & 14 & 5 \\
\hline $\begin{array}{l}\text { Panic disorder } \\
\text { without agoraphobia }\end{array}$ & 0 & 0 & 0 \\
\hline Agoraphobia & 0 & 0 & 0 \\
\hline \multicolumn{4}{|l|}{ Obsessive compulsive } \\
\hline \multicolumn{4}{|l|}{ Generalised anxiety } \\
\hline Social phobia & 0 & 8 & 7 \\
\hline \multicolumn{4}{|l|}{ HAM D } \\
\hline 07 & 86 & 67 & 65 \\
\hline $8 \quad 17$ & 14 & 16 & 14 \\
\hline $18 \quad 25$ & 0 & 14 & 18 \\
\hline$>25$ & 0 & 3 & 3 \\
\hline
\end{tabular}

suffering from moderate or severe ED did not have depressive symptoms.

Mild and moderate depressive symptoms made up 15\% in each group of respondents with moderate or severe ED. Those participants who had mild or moderate ED were more likely to suffer from premature ejaculation.

\section{Discussion}

The results of this study suggest that men suffering from ED are likely to have a co-morbid psychiatric disorder $142 \%, 95 \% \mathrm{Cl}$ : 32.2 - 52.3\%). Numerous studies have shown a high correlation between depression and ED, ${ }^{1,2}$ and this study supports these findings, with $33 \%$ of the participants having depressive symptoms on the HAM-D. This is higher than the $15 \%$ lifetime prevalence of major depressive disorder occurring in men. ${ }^{14}$ One of the difficulties experienced in this study was to find South African general population figures for depression and anxiety disorders for comparison, and therefore lifetime prevalence was used. The probability of depression seems to increase with the degree of ED. The co-morbidity of ED and depressive symptoms is also important as it affects compliance with treatment. Patients with ED and depressive symptoms are more likely to discontinue treatment for ED than patients with ED and no depressive symptoms.'

Twenty-one participants (21\%, 95\% Cl: $13.5-30.3 \%)$ were diagnosed with an anxiety disorder, which is slightly higher than the lifetime prevalence of anxiety disorder in men (19.2\%). ${ }^{15}$ When subdivided into the specific anxiety disorders none of the individual anxiety disorders was higher than the lifetime prevalence. Anxiety disorders were only diagnosed in participants with moderate to severe ED. Twelve respondents had both depressive symptoms and an anxiety disorder, showing that $36 \%$ of patients with depressive symptoms had a co-morbid anxiety disorder. In this study, the majority of men presenting with ED were between the ages of 30 and 59 years. Although the risk of ED increases with age, it seems that ED causes more anxiety in this age group than in those above the age of 59 years. ED may adversely affect quality of life and impair social and occupational health to a greater extent in this group of respondents. Although not supported in this study, the risk of substance abuse in this group of men might also be higher.

It was interesting to note that $51 \%$ of respondents in the severe ED group reported suffering from premature ejaculation, compared with $70 \%$ of respondents in the mild and moderate groups. Premature ejaculation is another major stressor for respondents, causing significant distress, performance anxiety and interpersonal conflict.

In accordance with the findings of the Massachusetts Male Aging Study, ${ }^{4}$ this study noted significant concomitant medical conditions. Participants diagnosed with moderate to severe ED were more likely to have an accompanying medical condition, most notably hypertension. The majority of the hypertensive sufferers were on antihypertensive medication, which is known to add to the problem of ED. 
As this study has shown, depressive and anxiety disorders and concomitant medical conditions are common in men presenting with ED. As ED is a multifactorial condition, and we are unable to determine what comes first (ED, depression or medical condition), the treating physician should screen ED patients for affective and anxiety disorders. The physician should also assess patients with affective and anxiety disorders for ED.

Ideally the assessment of ED should occur before the commencement of psychotropic medication. Patients should also be screened routinely for medical conditions, namely hypertension, diabetes mellitus, hypercholesterolaemia and cardiac conditions. The treatment regimen for medical and psychiatric conditions should be altered to avoid side-effects of certain medications compounding the problem of ED.

As previously mentioned, the MINI rating scale was problematic as the questions were direct and respondents gave numerous false-positive answers. To prevent a false diagnosis, non-directive questions were asked to confirm positive answers obtained from the MINI. Should the present study be repeated, alternative anxiety rating scales could be used.

\section{References}

1. Shabsigh R, Klein L, Seidman S, et al. Increased incidence of depressive symptoms in men with erectile dysfunction. J Urol 1998; 52: 848-852.

2. Stuart N, Seidman M. Exploring he relationship between depression and erectile dysfunction in aging men. J Clin Psychiatry 2002; 63 (5): 5-12.

3. Araujo A, Durante R, Feldman $H$, et al. The relationship between depressive symptoms and male erectile dysfunction: Cross-sectional results from he Massachusetts Male Aging Study. Psychosom Med 1998; 60: 458-465.

4. Araujo A, Johannes C, Feldman $H$, et al. Relation between psychosocial risk factorsand incident erectile dysfunction: prospective results from the Massachusetts Male Aging Study. Am J Epidemiol 2000; 152: 533-54 1.

5. Goldstein I. The mutually reinforcing triad of depressive symptoms, cardiovascular disease, and erectile dysfunction. Am J Cardiol 2000; 86(2A): 4 1F-45F.

6. Roose $\mathrm{S}$, Seidman S. Sexual activity and cardiac risk: is depression a contributing factor. Am J Cardiol 2000; 86(2A): 38F-40F.

7. Rosen RC, Lane RM, Menza M. Effects of SSRIs on sexual function: a critical review I Clin Psychopharmacol 1999; 19: 67-85

8. Muller $M$, Benkert $O$. Lower self-reported depression in patients wi h erectile dysfunction after treatment wi h sildenafil. J Affect Disord 2001; 66: 255-261.

9. Rosen R. Psychogenic erectile dysfunction. Urol Clin North Am 2001; 28 : 269 279.

10. Rosen RC, Cappelleri JC. Development and evaluation of an abridged 5 version of the International Index of Erectile Dysfunction (IIEF-5) as a diagnostic tool for erectile dysfunction. Int I Impot Res 1999; 11 : 319-326.

11. Kaplan H, Sadock B. Synopsis of Psychiatry. 8th ed. Maryland: Williams and Wilkins, 1997: 689

12. Hamilton M. A rating scale for depression. J Neurol Neurosurg Psychiatry 1960; 23: $56-62$.

13. Sheehan D. Mini International Neuropsychiatric Interview. Hospital de la Salpetriere, Paris, France; University of South Florida, College of Medicine, USA, 1992

14. Kaplan H, Sadock B. Synopsis of Psychiatry. 8th ed. Maryland: Williams and Wilkins, 1997: 538

15. Kaplan H, Sadock B. Synopsis of Psychiatry. 8th ed. Maryland: Williams and Wilkins, 1997: 583. 